嘕

Original

\title{
Respuesta reproductiva, metabólica y cambios corporales en ovejas alimentadas con dos niveles de energía
}

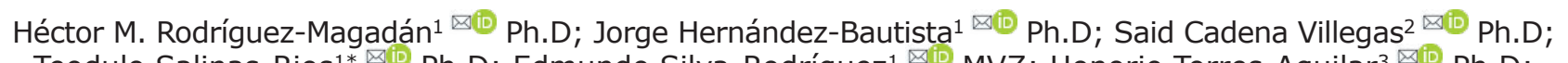

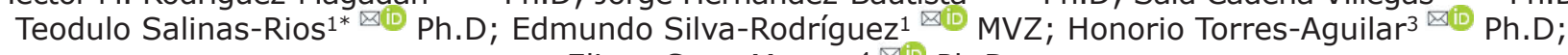
Eliseo Sosa-Montes ${ }^{\bowtie(0)}$ Ph.D.

\begin{abstract}
${ }^{1}$ Universidad Autónoma Benito Juárez de Oaxaca, Facultad de Medicina Veterinaria y Zootecnia. Oaxaca, México. ${ }^{2}$ Colegio de Postgraduados, Campus Tabasco; Periférico Carlos A. Molina. Tabasco, México.

3Universidad Autónoma Benito Juárez de Oaxaca, Facultad de Ciencias Químicas. Oaxaca, México.

${ }^{4}$ Universidad Autónoma Chapingo, Departamento de Zootecnia. Texcoco, Estado de México, México.

*Correspondencia: salinas980@hotmail.com
\end{abstract}

Recibido: Julio 2020; Aceptado: Enero 2021; Publicado: Mayo 2021.

\section{RESUMEN}

Objetivo. Evaluar la respuesta reproductiva, estado metabólico y cambios corporales en ovejas Dorper y Katahdin suplementadas con dos niveles de energía previo al empadre. Materiales y métodos. Se utilizaron 14 hembras Katahdin y 13 Dorper distribuidas en 2 tratamientos a las cuales se les sincronizó el celo. Catorce días previo a la sincronización de estros, las ovejas se distribuyeron en 2 tratamientos de acuerdo con el nivel de energía en la dieta, T1:2.0 y T2:2.5 Mcal kg-1 de EM. La alimentación abarcó 14 días de adaptación y 14 de alimentación con dieta integral. Al inicio y al final del estudio se midió el espesor de grasa dorsal, área del ojo de la costilla, peso corporal y concentraciones de glucosa e insulina. Se midieron los principales parámetros reproductivos como respuesta a estro, porcentaje de gestación y prolificidad. Se realizaron análisis de varianza y pruebas de ji cuadrada para determinar el efecto del nivel de energía y el genotipo. Resultados. El genotipo y el nivel de energía no afectaron ninguna de las variables medidas, aunque se encontró que con ambos niveles de energía existió un incremento en las concentraciones de glucosa, así como una ganancia en peso, grasa corporal y área del ojo de la costilla. Conclusiones. Debido a que no existió diferencia en los parámetros reproductivos y cambios corporales en ovejas al alimentarlas con los dos niveles de energía, es mejor la alimentación con $2.0 \mathrm{Mcal} \mathrm{kg}^{-1}$ de EM, ya que reduce costos de alimentación.

Palabras clave: Condición corporal; estro; fertilidad; flushing; grasa (Fuente: AIMS, MeSH).

\section{ABSTRACT}

Objective. To evaluate the reproductive response, metabolic state and body changes in Dorper and Katahdin ewes supplemented with two energy levels prior to insemination. Materials and methods. The animals used in this experiment were 14 Katahdin and 13 Dorper ewes distributed in two treatments, in which estrus had been synchronized. Fourteen days prior to synchronization

Como citar (Vancouver).

Rodríguez-Magadán HM, Hernández-Bautista J, Cadena-Villegas S, Salinas-Rios T, Silva-Rodríguez EA, Torres-Aguilar H, Sosa-Montes E. Respuesta reproductiva, metabólica y cambios corporales en ovejas alimentadas con dos niveles de energía. Rev MVZ Córdoba. 2021; 26(3):e2129. https://doi.org/10.21897/rmvz.2129 
of estrus, the ewes were distributed in two treatments according to the energy level of the diet, $\mathrm{T} 1: 2.0$ and T2:2.5 Mcal kg-1 of ME. Feeding consisted of 14 days of adaptation and 14 of feeding with integral diet. At the start and finish of each study, measurements were made of dorsal fat thickness, area of the rib-eye, body weight and glucose and insulin concentrations. The principal reproductive parameters were measured, such as response to estrus, percentage of gestation and prolificity. Analyses of variance were made along with ji-squared tests to determine the effect of the energy level and genotype. Results. The genotype and energy level did not affect any of the variables measured, although it was found that with both energy levels there was an increase in glucose concentrations, as well as an increase in weight, body fat and area of the rib-eye. Conclusions. Because there was no difference in the reproductive parameters and body changes with feeding in the two energy levels, it is preferable to use the feed with $2.0 \mathrm{Mcal} \mathrm{kg}-1$ of $\mathrm{ME}$, given that it reduces costs.

Keywords: Body condition; estrus; fat; fertility; flushing (Source: AIMS, MeSH).

\section{INTRODUCCIÓN}

Una adecuada alimentación es determinante para mejorar la respuesta reproductiva de los ovinos. Durante la lactancia, existe una pérdida de peso (1), sobre todo en aquellas ovejas que son alimentadas con bajos niveles de energía metabolizable (2). Una correcta suplementación alimenticia (3) mejora la condición corporal de las hembras y por consecuencia la respuesta reproductiva. Sin embargo, en la mayoría de los rebaños esta práctica no se realiza debido al desconocimiento de sus beneficios o a la falta de recursos económicos de los productores; por lo tanto, las ovejas llegan al destete con una baja condición corporal.

Lo deseable es que posterior al destete de las crías, las ovejas sean empadradas en un periodo corto; sin embargo, en ocasiones esto se dificulta debido a la baja condición corporal de la oveja. Se ha reportado que las ovejas con condición media tienen una mayor tasa de concepción que las de condición baja (4). Una alternativa es la suplementación previa al empadre, ya que se ha demostrado que mejora la respuesta reproductiva $(5,6)$; al respecto se ha demostrado que al suministrar dietas altas en energía por un periodo corto o largo incrementa la tasa ovulatoria de las ovejas (7). La mejora en los parámetros reproductivos va acompañada de cambios en concentración de glucosa e insulina en plasma sanguíneo (8).

Realizar flushing por periodos cortos con productores que no suplementan con concentrados en su sistema de producción se dificulta debido a que estas dietas se basan en el uso de granos para incrementar el aporte energético, lo cual requiere periodos de adaptación a la dieta para evitar problemas digestivos; por lo que, un flushing con un periodo de adaptación es viable en estos casos. Es importante que además de incrementar la respuesta reproductiva, las ovejas recuperen parte de su condición corporal, por lo tanto, la suplementación por un periodo previo al empadre podría ayudar (6).

Se ha demostrado que ovejas con una condición corporal menor de 2 en una escala de 1 a 5 tienen una menor tasa ovulatoria que las de condición mayor a 3 (9). Aunque existen recomendaciones de los requerimientos de las ovejas previo al empadre, sobre todo basadas en el peso (10), podrían existir diferencias debido al genotipo, clima del lugar y grasa corporal al momento del inicio de la suplementación, por lo que la condición corporal y el espesor de grasa dorsal al inicio de la sincronización de estros puede modificar la respuesta reproductiva. También es necesario considerar que en ocasiones o en algunos lugares, no existe disponibilidad de granos o alguna otra fuente energética, esto genera la necesidad de probar dietas con diferentes niveles de energía sin afectar los resultados del empadre.

En algunos estados de México se ha popularizado la introducción de sementales Dorper y Katahdin, logrando con ello tener ovejas de estas razas. Debido a todo lo anterior, el objetivo del presente estudio fue evaluar la respuesta reproductiva, el estado metabólico y los cambios corporales en ovejas Dorper y Katahdin suplementadas previo al empadre con dos niveles de energía.

\section{MATERIALES Y MÉTODOS}

Manejo de animales. Los animales fueron manejados de acuerdo con la norma oficial mexicana NOM-062-ZOO-1999, de 
especificaciones técnicas para la producción, cuidado y uso de animales de laboratorio.

Sitio de estudio. El estudio se realizó en la localidad de San Pedro Añañe, perteneciente al municipio de San Bartolo Soyaltepec, Oaxaca, México; ubicado a $17^{\circ} 31^{\prime} 15.5^{\prime \prime}$ latitud norte, $97^{\circ} 22^{\prime} 6^{\prime \prime}$ longitud oeste y a una altitud de 2189 $\mathrm{m}$. El clima es sub-húmedo con lluvias en verano.

Población de estudio y diseño experimental. Se utilizaron 13 ovejas Dorper y 14 Katahdin de 1 a 3 partos, con una media y desviación estándar de peso de $36.44 \pm 5.52 \mathrm{~kg}$, las cuales habían sido destetadas entre 2 y 3 semanas previo al inicio del estudio. Una vez destetadas se alimentaron con pacas de rastrojo de maíz y pasto Rhodes (Chloris gayana). La condición corporal fue de $3.08 \pm 0.09$ en una escala de 1 a 5. Las ovejas fueron alojadas individualmente y se distribuyeron en 2 tratamientos de acuerdo con el genotipo y condición corporal, de tal manera que ambos tratamientos quedaron equilibrados en cuanto a ovejas con diferente condición corporal. T1: ovejas alimentadas con una dieta integral con 2.0 Mcal kg-1 de EM (6 ovejas Dorper y 6 Katahdin) y T2: ovejas suplementadas con una dieta integral con $2.5 \mathrm{Mcal}_{\mathrm{kg}}{ }^{-1}$ de EM (7 ovejas Dorper y 8 Katahdin). El porcentaje de proteína cruda fue de $13 \%$ para ambas dietas (Tabla 1 ). La composición bromatológica de los ingredientes se realizó previo a la formulación de las dietas. El periodo de adaptación a la dieta inició con la presincronización de estros. Este periodo consistió en proporcionarle $200 \mathrm{~g}$ de alimento en la primer semana y $400 \mathrm{~g}$ en la segunda semana y rastrojo de maíz molido ( $3.06 \%$ PC, 0.97 Mcal de EM kg-1 y $32.95 \%$ de Nutrientes Digestibles Totales) a libre acceso. Desde el inicio de la sincronización de estros y hasta la inseminación artificial se le proporcionó $1.5 \mathrm{~kg}$ de dieta integral por oveja, para ello se proporcionó $700 \mathrm{~g}$ a las 8:00 h y $800 \mathrm{~g}$ a las $16: 00 \mathrm{~h}$, por lo que el periodo de suplementación consistió en 28 días (14 días de adaptación y 14 días de alimentación con dieta integral) (Figura 1).

Medición del estado corporal. El peso corporal, espesor de grasa dorsal y área del ojo de la costilla se midieron al inicio del periodo de adaptación a la dieta y al final de la alimentación (Figura 1). Para la medición de grasa y área del ojo de la costilla se utilizó un ultrasonido Pie medica ${ }^{\circledR}$, modelo Aquila, con transductor de 3.5 $\mathrm{MHz}$ y acoplador acústico.
Tabla 1. Ingredientes y composición química de dietas con 2 niveles de energía proporcionadas a las ovejas previo al empadre.

\begin{tabular}{ccc}
\hline Ingrediente & T1 & T2 \\
\hline Grano de maíz (\%) & 27.4 & 50.5 \\
Pasta de soya (\%) & 12.8 & 10 \\
Rastrojo de frijol (\%) & 25 & 14 \\
Rastrojo de maíz (\%) & 24 & 14.6 \\
Aceite de soya (\%) & 0 & 3 \\
Urea (\%) & 0.8 & 0.9 \\
Sal mineral (\%) & 2 & 2 \\
Sal común (NaCl) (\%) & 1 & 1 \\
Melaza (\%) & 7 & 4 \\
Composición química & & \\
Proteína Cruda (\%) & 13.0 & 13.0 \\
Mcal kg-1 de EM & 2.0 & 2.5 \\
Fibra Cruda (\%) & 16.98 & 10.68 \\
Costo por kg de alimento & 4.11 & 4.52 \\
(\$, pesos mexicanos) &
\end{tabular}

T1: ovejas suplementadas con $2.0 \mathrm{Mcal} \mathrm{kg}^{-1}$ de EM y T2: ovejas suplementadas con $2.5 \mathrm{Mcal}_{\mathrm{kg}}^{-1}$ de EM.

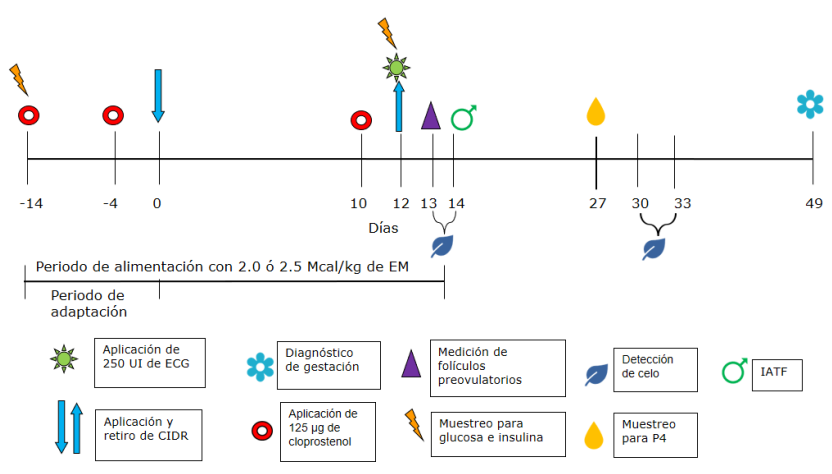

*IATF: Inseminación artificial a tiempo fijo

Figura 1. Protocolo de sincronización de estros y toma de muestras sanguíneas para medición de glucosa, insulina y progesterona en ovejas alimentadas con 2.0 y $2.5 \mathrm{Mcal} \mathrm{kg}^{-1}$ de EM previo al empadre.

Presincronización y manejo reproductivo. Previo a la sincronización de celo se aplicó una doble dosis de $125 \mu \mathrm{g}$ de cloprostenol con 8 días de intervalo, con la finalidad que todas las ovejas se encontraran en la misma fase del ciclo estral al momento del inicio del experimento. Seis días posteriores a la segunda aplicación se colocó un dispositivo intravaginal impregnado con 0.3 $\mathrm{g}$ de progesterona el cual permaneció por 12 días, dos días previos a su retiro se le aplicó vía intramuscular $125 \mu \mathrm{g}$ de cloprostenol. Al retiro del CIDR se aplicó 250 UI de eCG, iniciándose 
la detección de estros 24 horas después, con un intervalo de 6 horas hasta el día de la inseminación artificial.

A las 48 horas de retirado el dispositivo con progesterona se midió el número y tamaño de los folículos preovulatorios localizados en ambos ovarios. Para ello, se utilizó un ultrasonido EMP 2000 vet $^{\mathrm{MR}}$ con un transductor lineal de 5.0 $\mathrm{MHZ}$. La inseminación artificial se realizó por laparoscopia a tiempo fijo, 52 horas posteriores al retiro del dispositivo con progesterona.

El retorno a celo se realizó entre los días 16 a 19 post inseminación y el diagnóstico de gestación a los 35 días (Figura 1). Se registraron borregas paridas y el número de corderos nacidos.

Determinación de glucosa, insulina $y$ progesterona. Para medir la concentración de glucosa e insulina se tomó una muestra sanguínea de la vena yugular en tubo vacutainer $B D \AA$, al inicio de la presincronización que fue también el inicio del periodo de adaptación a la dieta y otra muestra al retiro del dispositivo con progesterona, posteriormente se centrifugó en una centrífuga VELAB modelo VE- $4000^{\circledR}$ a $1600 \mathrm{~g}$ por 10 minutos y el suero obtenido fue separado para ser ultracongelado a $-70^{\circ} \mathrm{C}$ en un equipo Thermo Scientific modelo 803CA hasta su análisis.

Trece días posteriores a la inseminación artificial se tomó otra muestra de sangre para la determinación de progesterona (P4). Esta fue tratada de manera similar que la obtenida para la medición de glucosa e insulina.

Para la determinación de glucosa se utilizó el reactivo "Glucose-SL assay" (DCL) y un analizador bioquímico automatizado ES-218 (Kontrol LAB). La cuantificación se realizó usando los métodos de punto final y de cinética, la lectura se realizó a una longitud de onda de $340 \mathrm{~nm}$. La concentración de insulina se realizó por medio de Elisa con el kit "insulin ELISA" de la marca Calbiotech. Los análisis de P4 se realizaron mediante ensayo inmunoenzimático (Immunometrics, UK Ltd, 280 Muster Road, London SW6 6BQ). La sensibilidad analítica fue de $0.13 \mathrm{ng} \mathrm{mL}^{-1}$ con coeficiente de variación intra e inter ensayo de 9.59 y $13.7 \%$, respectivamente.

Análisis estadístico. Para el análisis de la concentración de glucosa, insulina, P4, ganancia de peso, área del ojo de la costilla y espesor de grasa dorsal se realizaron análisis de varianza usando como efecto fijo el tratamiento, el genotipo y su interacción. Las variables respuesta a celo, retorno a celo, porcentaje de gestación a los 35 días y prolificidad se analizaron con una prueba de ji cuadrada, y la variable horas al inicio del estro con un análisis de supervivencia, utilizándose el paquete estadístico SAS versión 9 (11).

\section{RESULTADOS}

No existió efecto del genotipo Dorper o Katahdin y su interacción con el nivel de energía en las variables evaluadas, por lo que los resultados en los cuadros se presentan solamente por efecto del nivel de energía.

El nivel de energía no afectó ( $p>0.05)$ la ganancia de peso, el espesor de grasa y el área del ojo de la costilla durante la suplementación previo al empadre. En la tabla 2 se observa que, durante el tiempo de alimentación previo al empadre, las ovejas alimentadas con 2 y $2.5 \mathrm{Mcal} \mathrm{kg}^{-1}$ ganaron 3.68 y $4.21 \mathrm{~kg}$ de peso, 0.271 y $0.286 \mathrm{~cm}$ de espesor de grasa y 2.024 y $1.92 \mathrm{~cm}^{2}$ de área del ojo de la costilla, respectivamente.

Tabla 2. Media \pm error estándar de ganancia de peso, espesor de grasa dorsal y área del ojo de la costilla en ovejas Dorper y Katahdin alimentadas con 2 niveles de energía previo al empadre.

\begin{tabular}{ccc}
\hline Variable & T1 & T2 \\
\hline Peso inicial $(\mathrm{kg})$ & $36.44 \pm 1.82$ & $36.75 \pm 1.63$ \\
Peso final* $(\mathrm{kg})$ & $41.79 \pm 2.29$ & $42.1 \pm 2.05$ \\
Ganancia de peso $(\mathrm{kg})$ & $3.68 \pm 0.64$ & $4.21 \pm 0.57$ \\
Espesor de grasa inicial $(\mathrm{mm})$ & $1.79 \pm 0.10$ & $1.88 \pm 0.10$ \\
Espesor de grasa final $(\mathrm{mm})$ & $2.71 \pm 0.18$ & $2.86 \pm 0.17$ \\
Ganancia de espesor de & $0.97 \pm 0.17$ & $1.02 \pm 0.16$ \\
grasa $(\mathrm{mm})$ & $7.606 \pm 0.365$ & $7.592 \pm 0.310$ \\
AOC inicial $\left(\mathrm{cm}^{2}\right)$ & $10.089 \pm 0.456$ & $9.518 \pm 0.435$ \\
AOC final $\left(\mathrm{cm}^{2}\right)$ & $2.024 \pm 0.312$ & $1.92 \pm 0.298$ \\
\hline Ganancia de AOC $\left(\mathrm{cm}^{2}\right)$ &
\end{tabular}

* Peso al final del estudio o al empadre; AOC: área del ojo de la costilla; T1: ovejas suplementadas con 2.0 Mcal kg-1 de EM previo al empadre y T2: ovejas suplementadas con $2.5 \mathrm{Mcal} \mathrm{kg}^{-1}$ de EM.

No existió efecto $(p>0.05)$ de la alimentación previo al empadre con los dos niveles de energía (2 ó 2.5 Mcal $\mathrm{kg}^{-1}$ de EM) en las variables reproductivas como hora de entrada a celo posterior al retiro del progestágeno, porcentaje de gestación, prolificidad, número y tamaño de los folículos preovulatorios (Tabla 3). 
Tabla 3. Variables reproductivas en ovejas Dorper y Katahdin alimentadas previo al empadre con dos niveles de energía.

\begin{tabular}{|c|c|c|}
\hline Variable & T1 & T2 \\
\hline \multicolumn{3}{|l|}{ Entrada a celo $(\mathrm{h})$} \\
\hline 24 & 33.3 & 13.3 \\
\hline 30 & 16.7 & 20.0 \\
\hline 36 & 25.0 & 46.7 \\
\hline 42 & 25.0 & 20.0 \\
\hline Retorno a celo (\%) & 8.4 & 13.4 \\
\hline Porcentaje de gestación & 91.6 & 86.6 \\
\hline Prolificidad & 1.27 & 1.38 \\
\hline $\begin{array}{l}\text { Número de folículos } \\
\text { preovulatorios }\end{array}$ & $3 \pm 0.3$ & $3 \pm 0.3$ \\
\hline $\begin{array}{l}\text { Tamaño de folículos } \\
\text { preovulatorios }(\mathrm{mm})\end{array}$ & $5.17 \pm 0.22$ & $4.67 \pm 0.22$ \\
\hline
\end{tabular}

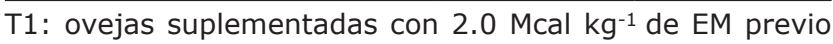
al empadre y T2: ovejas suplementadas con 2.5 Mcal kg-1 de EM.

Al comparar las concentraciones de progesterona en el día 13 post-inseminación y de glucosa e insulina al final de la alimentación con ambos niveles de energía, se encontró que no existió efecto ( $p>0.5$ ) de la suplementación con 2.0 ó 2.5 Mcal kg-1 de EM, genotipo e interacción (Tabla 4).

Tabla 4. Media \pm error estándar de las concentraciones de progesterona, glucosa e insulina en ovejas Dorper y Katahdin suplementadas con dos niveles de energía previo al empadre.

\begin{tabular}{ccc}
\hline Variable & T1 & T2 \\
\hline Progesterona $\mathrm{ng}(\mathrm{mL})$ & $8.67 \pm 1.67$ & $9.67 \pm 1.02$ \\
Glucosa inicial mmol/L & $3.56 \pm 0.07$ & $3.67 \pm 0.07$ \\
Glucosa final mmol/L & $4.75 \pm 0.35$ & $4.51 \pm 0.35$ \\
Insulina inicial pmol/L & $13.98 \pm 1.62$ & $17.62 \pm 2.95$ \\
Insulina final pmol/L & $13.06 \pm 0.84$ & $12.84 \pm 0.80$ \\
\hline
\end{tabular}

T1: ovejas suplementadas con $2.0 \mathrm{Mcal} \mathrm{kg}^{-1}$ previo al empadre y T2: ovejas suplementadas con $2.5 \mathrm{Mcal} \mathrm{kg}^{-1}$.

\section{DISCUSIÓN}

La alimentación con 2.0 ó $2.5 \mathrm{Mcal} \mathrm{kg}^{-1}$ de EM durante 14 días de adaptación y 14 días de alimentación con dieta integral previo al empadre en ovejas Dorper y Katahdin no modificó los parámetros reproductivos, las concentraciones de glucosa, insulina y progesterona, así como los cambios en peso y espesor de grasa dorsal.
Se han encontrado tasas de concepción similares entre ovejas Katahdin y Dorper (4); por otra parte, se han observado diferentes resultados en la respuesta reproductiva al suplementar con dietas energéticas previo al empadre. Se ha reportado que al suplementar al $1.5 \%$ del peso vivo por 35 días con un concentrado con 3.54 Mcal kg-1 de energía digestible a ovejas en pastoreo durante la estación reproductiva y con disponibilidad de forraje, no se afecta el número de cuerpos lúteos y la respuesta al estro (12). En un estudio posterior en condiciones similares, pero realizado en la estación de verano, considerada la época no reproductiva y con escasez de forraje, se encontró que la suplementación incrementó el porcentaje de ovejas ovuladas y el número de cuerpos lúteos (6); por tal motivo, la disponibilidad y cantidad de pasto se deben considerar al formular las raciones a utilizar para la suplementación. En el presente estudio la alimentación con dieta integral con 2 ó 2.5 Mcal kg-1 de EM previo a la inseminación artificial no afectó la respuesta reproductiva. Contrario a esto, se ha reportado que la alimentación con $3.0 \mathrm{Mcal} \mathrm{kg}^{-1}$ de ED por un periodo corto ( 5 días antes a 1 día después del retiro del progestágeno) o un periodo largo (15 días antes a 1 día después del retiro del progestágeno) incrementan el porcentaje de ovulaciones dobles (7).

Aunque la suplementación energética, ya sea por periodo largo de 16 días o corto de 6 días incrementan las ovulaciones dobles, es necesario no exceder el porcentaje de proteína en la dieta (13). El uso de plantas forrajeras de alta calidad nutritiva, son también una alternativa en los lugares donde hay disponibilidad, ya que mejora la tasa de ovulación (5). Por lo tanto, la buena alimentación previo al empadre ha demostrado mejorar los parámetros reproductivos. En el presente estudio no existió diferencia entre las dietas con 2.0 y $2.5 \mathrm{Mcal} \mathrm{kg}^{-1}$ de EM en ovejas Dorper y Katahdin, aunque los porcentajes de fertilidad encontrados son buenos. Sin embargo, si se quisiera incrementar más la prolificidad se podría probar con dietas con más de $2.5 \mathrm{Mcal}$ $\mathrm{kg}^{-1}$ de EM.

Dietas altas en energía ( $130 \%$ del requerimiento de energía de mantenimiento) por un periodo corto no tienen efecto en el número de folículos reclutados; sin embargo, se incrementa la duración del estro, el tamaño del folículo ovulatorio y la tasa de ovulación (14). En 
nuestro estudio, la alimentación con 2.0 ó 2.5 Mcal $\mathrm{kg}^{-1}$ de EM en ovejas Dorper y Katahdin, no afectó el número y tamaño de los folículos preovulatorios y la prolificidad; contrario a ello, en ovejas Ossimi se ha encontrado un incremento en el número de folículos medianos (3 a $5 \mathrm{~mm}$ ) y grandes ( $>$ a $5 \mathrm{~mm}$ ), así como en la ovulación al proporcionar una dieta con 2.6 Mcal $\mathrm{kg}^{-1}$ de EM con respecto a las alimentadas con $2.0 \mathrm{Mcal}_{\mathrm{kg}}{ }^{-1}$ de EM (15). Estas diferencias se pueden deber a múltiples factores, entre ellas los diferentes requerimientos nutricionales y diferente respuesta reproductiva de los genotipos o que la alimentación con $2.6 \mathrm{Mcal} \mathrm{kg}^{-1}$ de EM se proporcionó de manera intermitente en diferentes días del ciclo estral, mientras que en el presente estudio la suplementación se realizó por un periodo largo previo a la inseminación artificial. Es probable que a diferencia de lo reportado por otros autores donde se ha incrementado la tasa ovulatoria y el tamaño de los folículos, en el presente estudio al alimentar con una dieta al

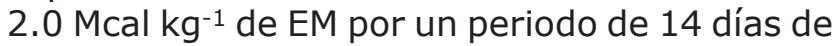
adaptación y 14 con dieta integral fue suficiente para incrementar las reservas corporales y las concentraciones de glucosa e insulina, las cuales están involucradas en el desarrollo de los folículos (16).

La condición corporal está relacionada con la cantidad de grasa en el cuerpo de las ovejas (17). Se ha reportado que la condición corporal influye en la respuesta reproductiva de las ovejas, donde se ha registrado una mayor tasa de concepción y número de corderos nacidos en ovejas al empadre con una condición corporal de entre 3 y 3.5 en contraste con aquellas con menor condición (18). De igual manera, se ha determinado que en ovejas Malpura la condición corporal óptima para empadre está entre 3.0 a 3.5, ya que la tasa de concepción y tamaño de camada disminuye con una condición menor o mayor (19), además al incrementar la condición corporal por arriba de 3.5 puede ser ineficiente la utilización de energía (17). En el presente estudio, las ovejas se distribuyeron de tal manera que ambos grupos quedaran balanceados de acuerdo a su condición corporal, al inicio del estudio se tenía una condición promedio de 3.0 y un espesor de grasa dorsal de $1.8 \mathrm{~mm}$ para las que consumieron 2.0 Mcal $\mathrm{kg}$ de EM y $1.88 \mathrm{~mm}$ para las que consumieron $2.5 \mathrm{Mcal}_{\mathrm{kg}}{ }^{-1}$ de EM. La alimentación con ambas dietas generó una recuperación en cuanto a músculo y grasa, por lo tanto, es probable que en caso de alimentar por un mayor periodo a las ovejas con $2.5 \mathrm{Mcal} \mathrm{kg}^{-1}$ de EM, hubieran ganado un mayor espesor de grasa y área del ojo de la costilla; sin embargo, el incrementar el estado corporal más allá de lo aceptado podría ocasionar efectos adversos en la reproducción.

En este estudio se utilizaron como medidas más exactas el espesor de grasa dorsal y el área del ojo de la costilla para medir el estado corporal de las ovejas, donde se observó que durante la suplementación por 14 días de adaptación y 14 de alimentación con dieta integral con 2.0 ó $2.5 \mathrm{Mcal} \mathrm{kg}^{-1}$ de EM, las ovejas incrementan su condición corporal y reservas de grasa.

En esquemas de suplementación por periodos largos previo al empadre, es más conveniente el uso de dietas con 2.0 Mcal kg de EM, ya que se demostró que se obtienen resultados reproductivos similares y a un menor costo por concepto de alimentación. Al incrementar el espesor de grasa dorsal, es probable que las ovejas también almacenaron grasa en diferentes partes del cuerpo, ya que se ha demostrado que existe una alta correlación positiva entre la grasa de la canal y la grasa interna, omental, mesentérica y pélvica (20). El espesor de grasa al momento del empadre no afecta la tasa de parición y el tamaño de camada; sin embargo, incrementa el peso de la camada al nacer y al destete (21); por lo que, además de considerar la importancia de la recuperación de la oveja al empadre en los parámetros reproductivos, es necesario valorar los beneficios en el desarrollo futuro de los corderos. Se han encontrado mejores parámetros productivos y reproductivos en ovejas con grasa corporal de 1.5 a $2 \mathrm{~mm}$ y las de más de $2 \mathrm{~mm}$ comparadas con aquellas con un espesor menor a $1.5 \mathrm{~mm}$ (22). En el presente estudio no hubiera sido posible hacer una clasificación de espesor de grasa tan baja al momento del empadre, ya que sólo el $8.6 \%$ de ovejas tuvieron un espesor menor a $2 \mathrm{~mm}$, concentrándose el $69.5 \%$ entre 2 y $3 \mathrm{~mm}$ de grasa.

La glucosa aporta energía a las células ováricas, mientras que la insulina regula su absorción (23). En respuesta a la deficiencia nutricional, se pueden generar cambios en las concentraciones de hormonas metabólicas, pudiéndose alterar el crecimiento folicular, la calidad de los ovocitos y la subsiguiente sobrevivencia del embrión. Se ha reportado que ovejas Île-de-France con una condición corporal menor a 2.0 tuvieron menor concentración de glucosa e insulina que las de mayor a 3.0, mientras que la suplementación la incrementa (9). También se ha reportado que, al incrementar la condición corporal debido a una 
buena nutrición, la concentración de insulina se incrementa al pasar de la condición corporal de 1.25 a 3.0, para posteriormente estabilizarse o tener un pequeño decremento al pasar de una condición de 3.0 a 4.0 (24). En el presente estudio a pesar que la grasa dorsal y el área del ojo de la costilla se incrementó durante la etapa de alimentación con 2 y $2.5 \mathrm{Mcal} \mathrm{kg}^{-1}$ de EM, la concentración de insulina no se incrementó, si no por el contrario existió un decrementó, el cual fue más marcado en el tratamiento con $2.5 \mathrm{Mcal}$, por lo que es posible que las ovejas en el presente estudio al tener una condición corporal promedio de 3.08 al inicio del estudio su concentración de insulina permaneció estable al proporcionarles las dietas.

El incremento en concentración de glucosa se da incluso con la suplementación por un periodo corto con dietas altas en energía (14). Tanto la cantidad de glucosa como la de insulina son rápidamente modificables, ya que un día después del inicio de la suplementación energética su concentración se incrementa (13). De igual manera se ha reportado que aparte de la glucosa también la insulina y leptina se incrementan en ovejas de pelo en pastoreo y suplementadas, pero esto no se ve reflejado en la fertilidad y prolificidad (25). Se ha encontrado que cuando existe restricción alimenticia se inhibe la foliculogénesis, tal vez por el decremento en la circulación de glucosa, insulina y glucagón, mientras que al suplementar este efecto es revertido y se incrementa el número de folículos mayores de $3.5 \mathrm{~mm}$ (8). Al final del presente estudio las concentraciones de glucosa e insulina no variaron entre las ovejas alimentadas con 2.0 y $2.5 \mathrm{Mcal} \mathrm{kg}^{-1}$ de EM, probablemente a que durante el tiempo largo que abarcó durante la adaptación y la alimentación con dieta integral, las ovejas incrementaron sus concentraciones, aumentaron la ganancia de peso, el espesor de grasa y área del ojo de la costilla en ambos tratamientos. Por lo que las ovejas lograron recuperarse al ser suplementadas por un periodo largo de tiempo aun con la suplementación con $2.0 \mathrm{Mcal} \mathrm{kg}^{-1}$ de EM.

La progesterona tiene un papel importante en evitar la luteólisis, ayudando con esto al reconocimiento materno de la preñez (26), mientras que el deterioro de la esteroidogénesis, - capacidad reducida de producción de progesterona conduce a la muerte de las células lúteas (27). Por lo que, la progesterona secretada por el cuerpo lúteo es crítica para el establecimiento y mantenimiento de la gestación temprana (28). El aporte extra de energía en la dieta de ovejas a través de la inclusión de $4 \%$ de aceite de palma en ovejas incrementa el metabolismo lipídico, incluyendo colesterol, lo que a su vez incrementa la concentración de progesterona; sin embargo, al incrementar a $6 \%$ la inclusión de aceite de palma no se modifica más el metabolismo lipídico y la concentración de progesterona (29), de igual manera en vacas con baja condición corporal en pastoreo y suplementadas con un concentrado con $4 \%$ de aceite de maíz se ha reportado que se incrementa la concentración de progesterona y colesterol. Por el contrario, en vacas lecheras se ha encontrado que la diferencia del $12 \%$ en el consumo de materia seca de forraje no altera el metabolismo de progesterona (30). En el presente estudio se agregó 3\% de aceite de soya en la dieta con 2.5 Mcal kg-1 de EM y se midió la concentración de progesterona en el día 13 post-inseminación ya que es cuando se da la caída natural de progesterona del ciclo estral. En ovejas gestantes el cuerpo lúteo debe producir suficiente progesterona para ayudar el reconocimiento materno de la gestación, al respecto, el suplementar previo al empadre con 2 ó 2,5 Mcal no influyó en la producción posterior de progesterona por el cuerpo lúteo.

Bajo las condiciones del presente estudio y debido a los resultados similares en las variables evaluadas y aun mayor costo de la dieta con 2.5 Mcal, se recomienda el uso de $2.0 \mathrm{Mcal} \mathrm{kg}^{-1} \mathrm{de}$ EM en ovejas previo al empadre.

Se concluye que la alimentación con una dieta con 2.0 ó 2.5 Mcal kg-1 de EM por un periodo de 14 días de adaptación y 14 de dieta integral previo al empadre en ovejas Dorper y Katahdin no afecta los parámetros reproductivos, estado metabólico y cambios corporales; sin embargo, con ambas dietas se incrementa el peso vivo y las reservas corporales, debido a ello y con la finalidad de reducir costos por alimentación se

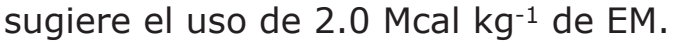

\section{Conflicto de interés}

Los autores declaran no tener conflicto de interés alguno relacionado a la publicación del presente artículo. 


\section{REFERENCIAS}

1. Naik MC, Krishnamoorthy $U$, Nagaraja R, Yathiraj S. Lactation pattern in mandya ewes and its influence o body weight gain in pre-weaning lambs. Indian Journal of Small Ruminants. 2016; 22(1):22-27. https://doi. org/10.5958/0973-9718.2016.00001.5

2. Castro FAB, Ribeiro ELA, Mitzubuti IY, Silva LDF, Barbosa MAA, Sousa CL, et al. Influence of pre and postnatal energy restriction on the productive performance of ewes and lambs. R Bras Zootec. 2012; 41(4):951-958. https://doi.org/10.1590/ $\underline{\mathrm{S} 1516-35982012000400017}$

3. Costa TJN, Marques RA, Torreão MCA, Rocha $B L$, Patricia GF, Jácome AM. Concentrate supplementation during pregnancy and lactation of ewes affects the growth rate of lambs from a variety of crosses. R Bras Zootec. 2014; 43(10):544-550. https://doi. org/10.1590/S1516-35982014001000006

4. Aké-Villanueva JR, Aké-López JR, SeguraCorrea JC, Magaña-Monforte JG, AkéVillanueva NY. Factors affecting conception rate of hair ewes after laparoscopic insemination with chilled semen under tropical conditions. Small Ruminant Res. 2017; 153:114-117. https://doi. org/10.1016/j.smallrumres.2017.06.006

5. King BJ, Robertson SM, Wilkins JF, Friend MA. Short-term grazing of lucerne and chicory increases ovulation rate in synchronised Merino ewes. Anim Reprod Sci. 2010; 121:242-248. https://doi.org/10.1016/j. anireprosci.2010.06.007

6. Naqvi SM, Sejian V, Karim SA. Effect of feed flushing during summer season on growth, reproductive performance and blood metabolites in Malpura ewes under semiarid tropical environment. Trop Anim Health Prod. 2013; 45(1):143-148. https:// doi.org/10.1007/s11250-012-0185-2

7. Habibizad J, Riasi A, Kohram H, Rahmani HR. Effect of long-term supplementation of high energy or high energy-protein diets on ovarian follicles and blood metabolites and hormones in ewes. Small Ruminant Res. 2015; 132:37-43. https://doi. org/10.1016/j.smallrumres.2015.10.004
8. Ying $S$, Wang $Z$, Wang $C$, Nie $H$, He D, Jia R, et al. Effect of diferent levels of short-term feed intake on folliculogenesis and folicular fluid and plasma concentrations of lactate dehydrogenase, glucose, and hormones in $\mathrm{Hu}$ sheep during the luteal phase. 2011. Reproduction. 2011; 142:699-710. https:// doi.org/10.1530/REP-11-0229

9. Scaramuzzi RJ, Oujagir L, Menassol JB, Freret $S$, Piezel A, Brown HM, et al. The pattern of LH secretion and the ovarian response to the 'ram effect' in the anoestrous ewe is influenced by body condition but not by short-term nutritional supplementation. Reprod Fert Develop. 2014; 26:1154-1165. https://doi.org/10.1071/RD13139

10. NRC (National Research Council). Nutrient Requirements of Small Ruminant. Sheep, goats, and new world cervids. Washington, D.C. USA. The National Academics Press. 2007. 246-247.

11. SAS. User's guide Statistics. Version 9.0 Cary: SAS Institute Inc., 2002.

12. Naqvi SMK, Soren NM, Karim SA. Effect of concentrate supplementation on performance, ovarian response, and some biochemical profile of Malpura ewes. Trop Anim Health Prod. 2011; 43:905-913. https://doi.org/10.1007/ s11250-011-9782-8

13. Habibizad J, Riasi A, Kohram H, Reza RR. Effect of feeding greater amounts of dietary energy for a short-term with or without eCG injection on reproductive performance, serum metabolites and hormones in ewes. Anim Reprod Sci. 2015; 160:82-89. https:// doi.org/10.1016/j.anireprosci.2015.07.007

14. Senosy W, Mahmoud GB, Abdel-Raheem ShM. Influence of short-term energy supplementation on estrus, ovarian activity and blood biochemistry in Ossimi ewes synchronized with fluorogestone acetate in the subtropics. Theriogenology. 2017; 88:152-157. https://doi.org/10.1016/j. theriogenology.2016.09.027 
15. Mahmoud GB, Abdel-Raheem ShM, Senosy W, Derar RI. Impact of a short periodenergy supplementation on the ovarian follicular dynamics, blood metabolites and sex hormones in ewes. J Dev Agric Econ. 2012; 1(6):145-152. http:// academeresearchjournals.org/journal/jaed/ archive/december-2012-vol.-1-(6)

16. Scaramuzzi RJ, Zouaïdi N, Menassol JB, Dupont J. The effects of intravenous, glucose versus saline on ovarian follicles and their levels of some mediators of insulin signalling. Reprod Biol Endocrin. 2015; 13:6. https://doi.org/10.1186/1477-7827$\underline{13-6}$

17. Morel PCH, Schreurs NM, Corner-Thomas RA, Greer AW, Jenkinson CMC, Ridler AL, et al. Live weight and body composition associated with an increase in body condition score of mature ewes and the relationship to dietary energy requirements. Small Ruminant Res. 2016; 143:8-14. https://doi.org/10.1016/j. smallrumres.2016.08.014

18. Vatankhah M, Talebi MA, Zamani F. Relationship between ewe body condition score (BCS) at mating and reproductive and productive traits in Lori-Bakhtiari sheep. Small Ruminant Res. 2012; 106:105-109. https://doi.org/10.1016/j. smallrumres.2012.02.004

19. Sejian V, Maurya VP, Naqvi SMK, Kumar D, Joshi $A$. Effect of induced body condition score differences on physiological response, productive and reproductive performance of Malpura ewes kept in a hot, semiarid environment. J Anim Physiol Anim Nutr. 2010; 94(2):154-161. https://doi. org/10.1111/j.1439-0396.2008.00896.X

20. Chay-Canul AJ, Ayala-Burgos AJ, Ku-Vera JC, Magaña-Monforte JG, Tedeschi LO. The effects of metabolizable energy intake on body fat depots of adult Pelibuey ewes fed roughage diets under tropical conditions. Trop Anim Health Prod. 2011; 43:929-936. https://doi. org/10.1007/s11250-011-9785-5

21. Petacek M, Duchacek J, Stadnik, Beran J, Stolc I. Effects of Ewes' live weight and backfat thickness at mating on fertility and production performance in suffolk sheep and their crosses. Bulg J Agric Sci. 2014; 20(5):1261-1267. https:// journal.agrojournal.org/page/en/details. php?article id=756
22. Abdel-Mageed II, El-Maaty AM. The effect of backfat thickness at mating on the reproductive and productive performances of ewes. Small Ruminant Res. 2012; 105:148-153. https://doi.org/10.1016/j. smallrumres.2011.11.022

23. Dupont J, Scaramuzzi RJ. Insulin signalling and glucose transport in the ovary and ovarian function during the ovarian cycle. Biochem J. 2016; 473:1483-1501. https:// doi.org/10.1042/BCJ20160124

24. Caldeira RM, Belo AT, Santos CC, Vazques MI, Portugal AV. The effect of body condition score on blood metabolites and hormonal profile in ewes. Small Ruminant Res. 2007; 68:233-241. https://doi.org/10.1016/j. smallrumres.2005.08.027

25. Catunda AGV, Lima ICS, Bandeira GC, Gadelha CRF, Pereira ES, Salmito-Vanderley CSB, et al. Blood leptin, insulin and glucose concentration in hair sheep raides in a tropical climate. Small Ruminant Res. 2013; 114:272-279. https://doi.org/10.1016/j. smallrumres.2013.07.008

26. Raheem KA. An insight into maternal recognition of pregnancy in mammalian species. J Saudi Soc Agric Sci. 2015; 16(1):1-6. https://doi.org/10.1016/j. jssas.2015.01.002

27. Tomać J, Cekinović $Đ$, Arapovic J. Biology of the Corpus luteum. Periodicum Biologorum. 2011; 113(1):43-49. https://hrcak.srce. $\underline{\mathrm{hr} / 67235}$

28. Lonergan $\mathrm{P}$, Forde $\mathrm{N}$, Spencer $\mathrm{T}$. Role of progesterone in embryo development in cattle. Reprod Fert Develop. 2016; 28:6674. https://doi.org/10.1071/RD15326

29. Bianchi $A E$, Macedo VP, Franca RT, Lopes STA, Lopes LS, Stefani LM. Effect of adding palm oil to the diet of dairy sheep on milk production and composition, function of liver and kidney, and the concentration of cholesterol, triglycerides and progesterone in blood serum. Small Ruminant Res. 2014; 117:78-83. https://doi.org/10.1016/j. smallrumres.2013.12.025

30. Hutchinson IA, Dewhurst RJ, Evans ACO, Lonergan P, Butler ST. Effect of grass dry matter intake and fat supplementation on progesterone metabolism in lactating dairy cows. Theriogenology. 2012; 78:878-886. https://doi.org/10.1016/j. theriogenology.2012.04.001 